\title{
The Effect of Number and Position of Tuck Stitches within the Pattern Repeat on Air Permeability of Weft-knitted Fabrics from Glass Yarn
}

\author{
Mehmet Erdem İnce \\ Faculty of Engineering, Department of Textile Engineering Gaziantep University, Turkey
}

\begin{abstract}
The fact that weft knitted fabrics has a stretchable, 3D, porous and interlocking structure makes them unique when manufactured from high performance fibers. Knitted fabrics with different architectures exhibit different properties. Different loop forms like tuck and skip stitches with various loop lengths reveal different physical and mechanical properties. Literature review indicated that wisely arrangement of tuck stitches within the pattern repeat alter the weft-knitted fabric structure from natural and synthetic fibers. Therefore, we studied the effect of number and location of tuck stiches on air permeability of weft-knitted fabrics from glass yarn. Single-bed, flat weft knitting machine was used to knit fabrics with different architectures from three-ply glass yarn. The nominal single-end count of used E-glass yarn was 136 tex. The number and location of tuck stitches within knit pattern affected significantly the thickness and the air permeability of weft-knitted fabrics from glass yarn.
\end{abstract}

Key words: glass yarn, weft knitted fabric, knit pattern, tuck stich, air permeability

\section{Introduction}

Weft knitting is a fast, low-cost and flexible fabric manufacturing technique. Intricate interior structure of weft knitted fabrics formed by interlinked stitches render them exceptional within the fabric family. They are stretchable, and easy-to-shape that distinguish them from woven and nonwoven fabrics. A needle of weft knitting machine can form loop, tuck or skip stitches with varying dimensions that allows manufacture of numerous knit designs, and design governs physical properties and performances of the resultant fabric. This study is about the effect of number and position of tuck stitches within the pattern repeat on air permeability of weft-knitted fabrics from glass. The literature review within this context is given below.

Mikucioniene et al. [1-3] studied the association between air permeability and flammability of knit fabrics as function of loop length, linear density of yarns and tightness factor. Their object was to optimize the safety and comfort (breathability, lightness and ventilation of fire-fighters). Thicker yarns filled the gaps within fabric and lowered air permeability; while increasing fabric burning time. The researcher obtained a high level correlation between burning time and tightness of the fabric.

Asif et al. [4] examined the effects of knit architecture on dimensional stability, spirality, fabric width, areal density, pilling resistance and color fastness to washing/light/rubbing of weft-knitted fabrics. Due to yarn robbing of tuck stitches from neighboring loops; presence of tuck stitches increased the thickness of the fabric that enhanced fabric areal density. Number of tuck stitches

Corresponding author: Address: Faculty of Engineering, Department of Textile Engineering Gaziantep University, 27310, Gaziantep TURKEY. E-mail address: eince@gantep.edu.tr, Phone: +903423172730 
improved resistance to pilling, width shrinkage, fabric diameters; and lowered spirality, length shrinkage. Color fastness to washing/light/rubbing was not affected by knitted structure as long as dyeing and finishing parameters stayed same.

Rashed and Mahamudul [5] investigated the effect of tuck stitch on bursting strength of single jersey fabrics from 30/s and 40/s Ne carded yarns. The researcher found that bursting strength was not directly influenced by percentage of tuck stitches; it changed as a function of sequence of tuck stitches in the same wale or course.

Kane et al. [6] compared dimensional, comfort, and mechanical properties of tuck-knit and single jersey fabrics from ring and compact cotton yarns. They also varied loop length. Fabric architectures were designated as single jersey (plain), single pique, double pique, honeycomb. Loop length changed dimensional, comfort, mechanical, and handle properties of fabric at statistically significant level. Tuck-knit stitch fabrics showed better abrasion resistance, air permeability, water absorbency, thermal insulation, mechanical performance, and total handle values than single jersey fabrics

Uyanık et al. [7, 8] studied the effects of number and location of tuck stitches on properties of weft knitted fabrics produced from cotton yarn. Presence of tuck stitches increased fabric thickness, areal density, width; and created more permeable (porous) fabric structures than fabrics from wholly loop stitches. Stitch length decreased bursting strength. Location of tuck stitches exhibited more pronouncing effect on bursting strength than number of tuck stitches.

Literature review indicated that knit architecture determines the properties of weft knitted fabrics from natural and synthetic yarns. Apparently, no attention was devoted so far to examining physical properties and performance of weft knitted fabrics from glass yarn. To the best of our knowledge, previous researches were not evaluated the effect of number and position of tuck stitches on air permeability of weft knitted fabrics from glass yarn. The aim of this paper is to reveal the effect of number and position of tuck stitches on air permeability of weft-knitted fabrics from glass yarn.

\section{Materials and Method}

In this study three-ply E-glass filament yarn with single-end yarn count of 136 tex was used to manufacture fabrics on Brother KH-864 manual, flat weft knitting machine with a fineness of 5 gauge.

Four different fabric architectures each representing a different number and arrangement of tuck stitches were considered. Single jersey fabric with completely loop stitches was also considered as control sample. These five different arrangements of the sample fabrics were given as schematically in Table 1. Also, the images of the sample fabrics on the knitting machine were given in Figure 1. Thickness and air permeability of weft knitted fabrics were measured. 
Table 1. Technical notations of the weft knitted fabrics

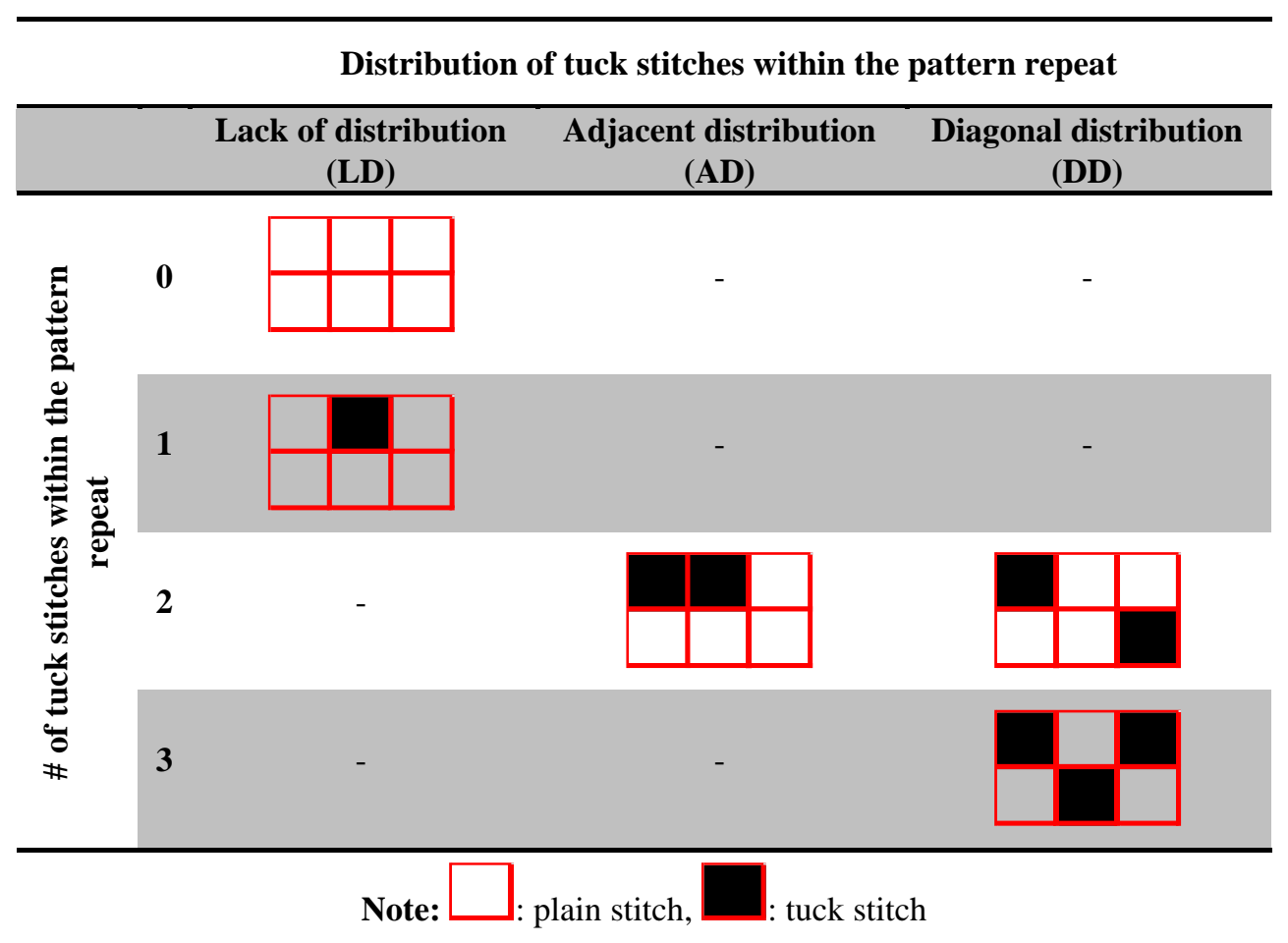

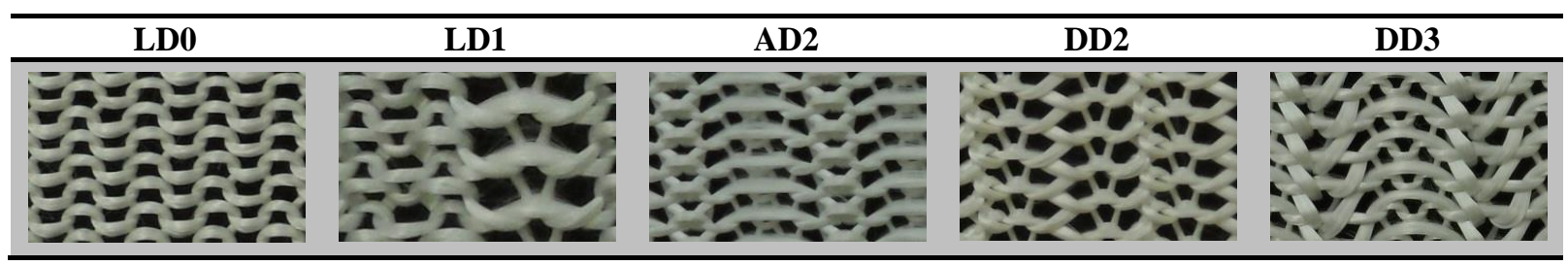

Figure 1. The images of the sample fabrics on the knitting machine

Thicknesses of weft knitted fabrics were measured by digital thickness gauge with a presser foot diameter of $21.15 \mathrm{~mm}$ and under a compaction pressure of $2 \mathrm{kPa}$. SDL ATLAS M021A was used to measure air permeability of the samples according to ISO 9237:1995. The pressure drop and the test head area were selected as $100 \mathrm{~Pa}, 20 \mathrm{~cm}^{2}$, respectively. Readings were recorded $\mathrm{cm}^{3} /\left(\mathrm{cm}^{2} \times \mathrm{s}\right)$ unit.

\section{Results and discussion}

\section{The effect of number of tuck stitches within the knit repeat on fabric thickness}

As seen in Figure 2 and Table 2, the fabric thickness increased significantly with the increase in the number of tuck stitches in the repeat of knitting. The presence of the tuck stitch also increased the thickness variation. The presence of the tuck stitch has shortened the fabric in both width and length direction, causing the fabric to increase in thickness. 


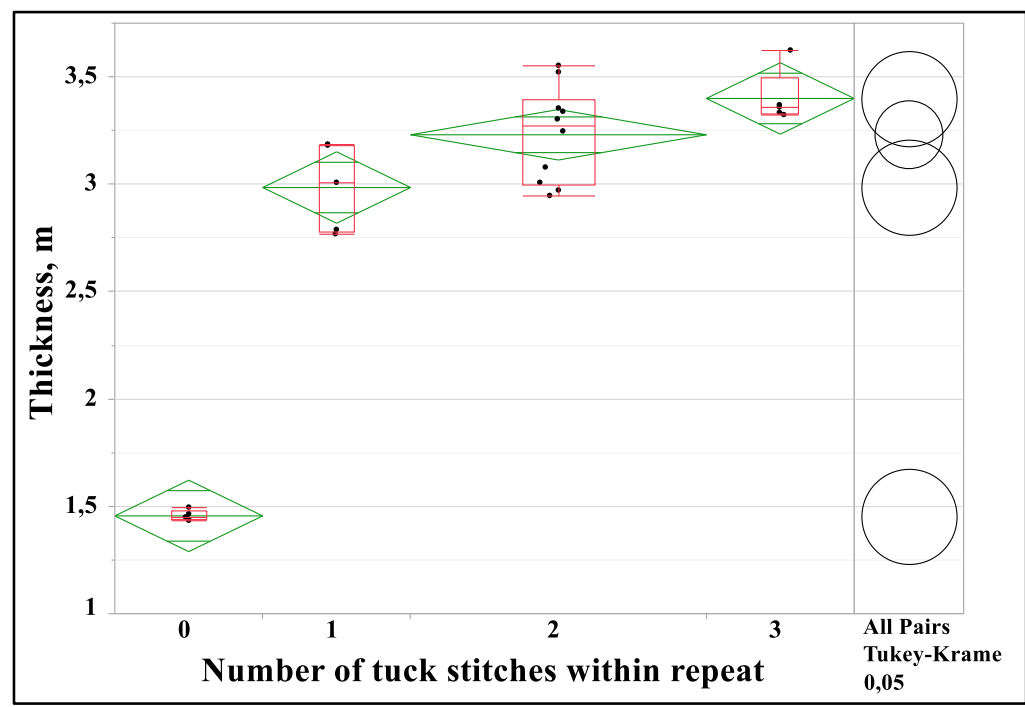

Figure 2. The effect of number of tuck stitches within knit repeat on fabric thickness

Note: The distance between the top and bottom edges of each green diamond represents the $95 \%$ confidence interval that is formed for the thicknesses of related number of tuck stitch level. One comparison circle per mean thickness of each number of tuck stitch level is given in the right columns. The comparison circles that represent number of tuck stitch levels with different mean thicknesses either do not intersect or intersect slightly. The height of the red box - a quantitative indication of variation - is called as "interquartile range".

Table 2. The effect of number of tuck stitches within knit repeat on fabric thickness

\begin{tabular}{|c|c|c|c|c|c|c|c|c|c|}
\hline Variable & Levels & & & $\bar{n}$ & mean & sd & $\mathbf{L L}$ & UL & p-value \\
\hline \multirow{4}{*}{$\begin{array}{l}\text { Number of tuck } \\
\text { stitches within knit } \\
\text { repeat }\end{array}$} & 3 & $\mathrm{~A}$ & & 5 & 3.40 & 0.12 & 3.24 & 3.56 & \multirow{4}{*}{$<0.0001$} \\
\hline & 2 & A B & & 10 & 3.23 & 0.22 & 3.07 & 3.39 & \\
\hline & 1 & B & & 5 & 2.98 & 0.20 & 2.73 & 3.24 & \\
\hline & 0 & & C & 5 & 1.46 & 0.02 & 1.43 & 1.48 & \\
\hline
\end{tabular}

Note: The number tuck stitch levels those are not connected by the same capital letter are statistically significant from each other under a significance level $(\alpha)$ adoption of 0.05 . n: number of observation, sd: standard deviation, LL: lower limit, UL: upper limit. The limits were established based on a confidence level of $95 \%$. The red color p-value indicates that the difference between at least two levels is statistically significant.

\section{The effect of distribution of tuck stitches on fabric thickness}

The fabrics in which the tuck stitches are located adjacent exhibited a higher fabric thickness than the fabrics in which they were placed diagonally (Figure 3 and Table 3). However, this difference did not reach a statistically significant level. The fact that the yarn forming the adjacent tuck stitches can move freely in the fabric plane has increased the fabric thickness. 


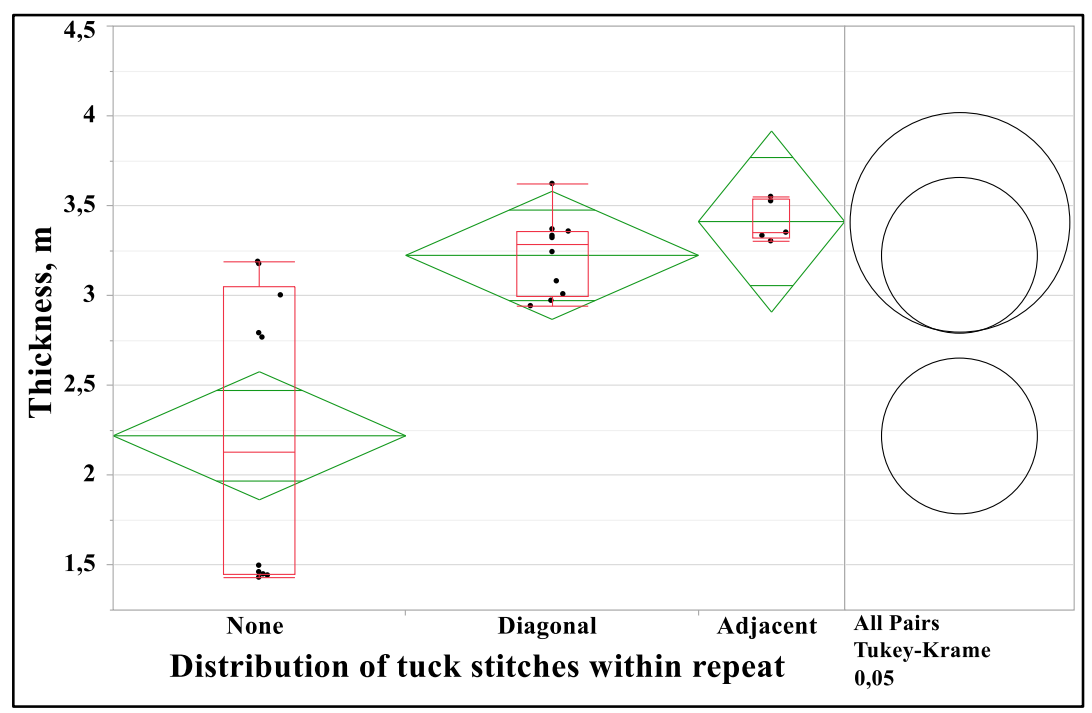

Figure 3. The effect of distribution of tuck stitches within knit repeat on fabric thickness

Table 3. The effect of distribution of tuck stitches within knit repeat on fabric thickness

\begin{tabular}{|c|c|c|c|c|c|c|c|c|}
\hline Variable & Levels & & $\mathrm{n}$ & mean & sd & $\overline{\mathbf{L L}}$ & $\mathbf{U L}$ & p-value \\
\hline \multirow{3}{*}{$\begin{array}{l}\text { Distribution of tuck } \\
\text { stitches within knit } \\
\text { repeat }\end{array}$} & Adjacent & $\bar{A}$ & 5 & 3.41 & 0.12 & 3.27 & 3.56 & \multirow{3}{*}{0.0003} \\
\hline & Diagonal & A & 10 & 3.22 & 0.22 & 3.07 & 3.38 & \\
\hline & None & & 10 & 2.22 & 0.82 & 1.64 & 2.80 & \\
\hline
\end{tabular}

\section{The effect of number of tuck stitches on fabric air permeability}

The insertion of only one tuck stitch into the knit repeat increased the air permeability of the fabric to a statistically insignificant level as compared with the plain knit of completely plain stitches. However, the insertion of two or three tuck stitches into the repeat of the knitting significantly reduced the air permeability of the fabric (Figure 4, Table 4). While the tuck stitch alone increased the porosity of the fabric, the two or three tuck stitches narrowed the fabric in the width and length direction, resulting in a firmer fabric formation that did not allow air to pass through. 


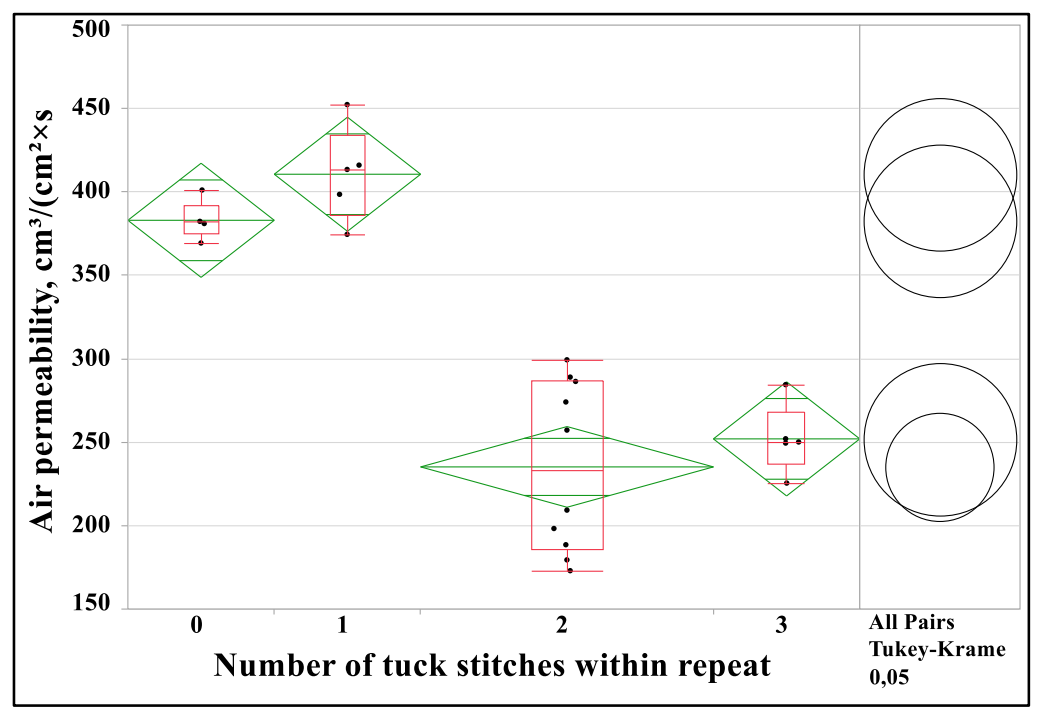

Figure 4. The effect of number of tuck stitches on air permeability of fabric

Table 4. The effect of number of tuck stitches on air permeability of fabric

\begin{tabular}{|c|c|c|c|c|c|c|c|c|c|}
\hline Variable & Levels & & & n & mean & sd & $\mathbf{L L}$ & $\mathbf{U L}$ & p-value \\
\hline \multirow{4}{*}{$\begin{array}{l}\text { Number of tuck } \\
\text { stitches within knit } \\
\text { repeat }\end{array}$} & 1 & A & & 5 & 410.60 & 28.49 & 375.22 & 445.98 & \multirow{4}{*}{$<0.0001$} \\
\hline & 0 & A & & 5 & 383.00 & 11.47 & 368.76 & 397.24 & \\
\hline & 3 & & B & 5 & 252.00 & 21.01 & 225.91 & 278.09 & \\
\hline & 2 & & B & 10 & 235.20 & 50.39 & 199.15 & 271.25 & \\
\hline
\end{tabular}

\section{The effect of distribution of tuck stitches on fabric air permeability}

Adjacent or diagonal placement of the tuck stitches in the knit repeat had a statistically significant effect on fabric air permeability (Figure 5, Table 5). The adjacent arrangement of the tuck stitches narrows the fabric in the width direction to form the tightest fabric structure.

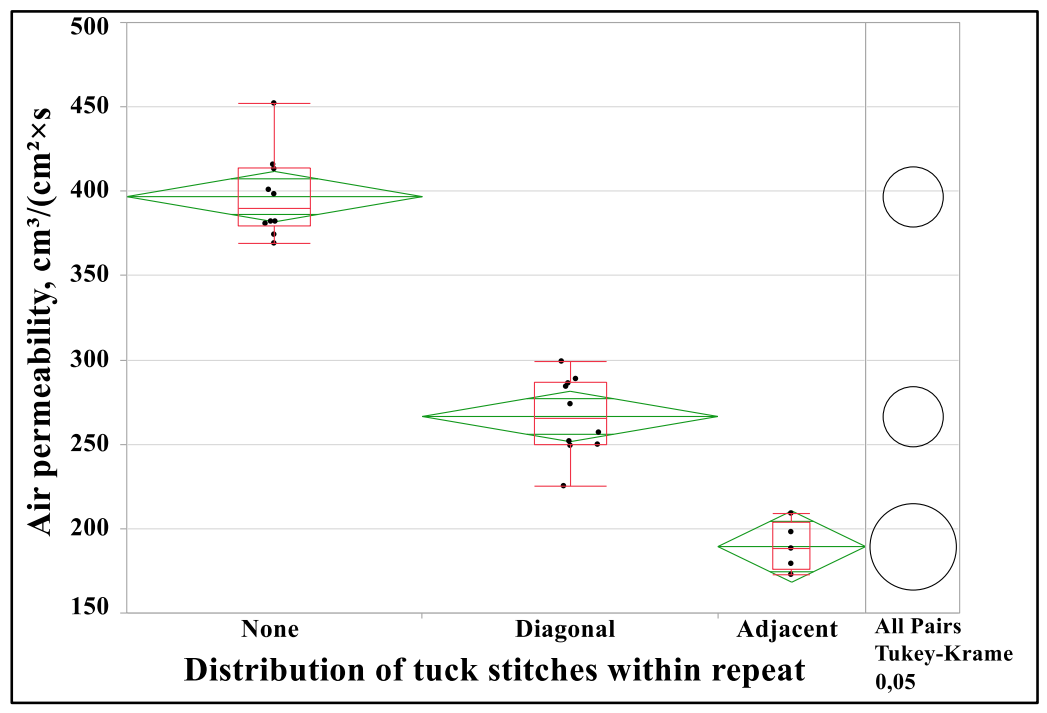

Figure 5. The effect of distribution of tuck stitches on air permeability of fabric 
Table 5. The effect of distribution of tuck stitches on air permeability of fabric

\begin{tabular}{|c|c|c|c|c|c|c|c|c|c|}
\hline Variable & Levels & & & n & mean & sd & $\mathbf{L L}$ & UL & p-value \\
\hline \multirow{3}{*}{$\begin{array}{l}\text { Distribution of tuck } \\
\text { stitches within knit } \\
\text { repeat }\end{array}$} & None & A & & 10 & 396.80 & 25.12 & 378.83 & 414.77 & \multirow{3}{*}{$<0.0001$} \\
\hline & Diagonal & B & & 10 & 266.50 & 23.35 & 249.80 & 283.20 & \\
\hline & Adjacent & & & 5 & 189.40 & 14.47 & 171.44 & 207.36 & \\
\hline
\end{tabular}

\section{Conclusions}

In this study, the effect of number and arrangement of tuck stiches in weft-knitted fabrics from glass yarn on thickness and air permeability were investigated by using five different knit patterns. Presence and arrangement of tuck stiches increased the thickness and lowered the air permeability of fabrics as compared with control sample of single jersey fabric. The presence of tuck stitches narrows the fabric in its width and length direction to form a thicker and less permeable fabric structure than that of a fabric with completely plain stitches. This study clearly demonstrated that the thickness and air permeability of fabrics made of glass fiber can be controlled as a result of the strategic placement of the tuck stitches in the knit repeat.

\section{References}

[1] Mikučionienė D, Baltušnikaitė J, Milašius R. Influence of the number of yarns in a loop on the flammability of knits. FIBRES \& TEXTILES in Eastern Europe 2011;19:71-4.

[2] Mikučionienė D, Milašiūtė L, Baltušnikaitė J, Milašius R. Influence of plain knits structure on flammability and air permeability. FIBRES \& TEXTILES in Eastern Europe

2012;20:66-9.

[3] Mikučionienè D, Milašiūtė L, Milašius R. Influence of knits structure on flammability and comfortability. AUTEX Research J 2014;14:226-32.

[4] Asif A, Rahman M, Farha FI. Effect of knitted structure on the properties of knitted fabric. Int J of Sci and Research (IJSR) 2015;4:1231-5.

[5] Md. Mahamudul R. Effect of tuck loop in bursting strength of single jersey knitted fabrics. IJRET: International J of Research in Engineering and Technology 2014;3:712-9.

[6] Kane CD, Patil UJ, Sudhakar P. Studies on the influence of knit structure and stitch length on ring and compact yarn single jersey fabric properties. Textile Research J 2007;77:57282.

[7] Uyanik S, Değirmenci Z, Topalbekiroğlu M, Geyik F. Examining the relation between the number and location of tuck stitches and bursting strength in circular knitted fabrics. FIBRES \& TEXTILES in Eastern Europe 2016;24:114-19.

[8] Uyanık S, Topalbekiroğlu M. The effect of knit structures with tuck stitches on fabric properties and pilling resistance. J of the Textile Institute 2017;108:1584-9. 